\title{
CAMA
}

Centre for Applied Macroeconomic Analysis

\section{Historical Decompositions for Nonlinear Vector Autoregression Models}

\section{CAMA Working Paper 62/2017 October 2017}

\section{Benjamin Wong}

Reserve Bank of New Zealand and

Centre for Applied Macroeconomic Analysis, ANU

\section{Abstract}

The historical decomposition is standard within the vector autogression (VAR) toolkit. It provides an interpretation of historical fluctuations in the modelled time series through the lens of the identified structural shocks. The proliferation of nonlinear VAR models naturally leads to extending the historical decomposition into nonlinear settings. This article discusses how to calculate an exact historical decomposition for a large class of popular nonlinear VAR models. In particular, the standard historical decomposition one obtains from a linear VAR is nested within the nonlinear case. The approach discussed in this article is sufficiently general to be relevant for many popular variants of nonlinear VAR models. 


\title{
Keywords
}

Historical Decomposition, Innovation Accounting, Nonlinear VAR models

\author{
JEL Classification
}

C32

\section{Address for correspondence:}

(E) cama.admin@anu.edu.au

\section{ISSN 2206-0332}

The Centre for Applied Macroeconomic Analysis in the Crawford School of Public Policy has been established to build strong links between professional macroeconomists. It provides a forum for quality macroeconomic research and discussion of policy issues between academia, government and the private sector.

The Crawford School of Public Policy is the Australian National University's public policy school, serving and influencing Australia, Asia and the Pacific through advanced policy research, graduate and executive education, and policy impact. 


\title{
Historical Decompositions for Nonlinear Vector Autoregression Models
}

\author{
Benjamin Wong* \\ Reserve Bank of New Zealand and Centre for Applied Macroeconomic \\ Analysis ${ }^{\dagger}$
}

\begin{abstract}
The historical decomposition is standard within the vector autogression (VAR) toolkit. It provides an interpretation of historical fluctuations in the modelled time series through the lens of the identified structural shocks. The proliferation of nonlinear VAR models naturally leads to extending the historical decomposition into nonlinear settings. This article discusses how to calculate an exact historical decomposition for a large class of popular nonlinear VAR models. In particular, the standard historical decomposition one obtains from a linear VAR is nested within the nonlinear case. The approach discussed in this article is sufficiently general to be relevant for many popular variants of nonlinear VAR models.
\end{abstract}

JEL Classification: C32

Keywords: Historical Decomposition, Innovation Accounting, Nonlinear VAR models

${ }^{*}$ The views do not necessarily reflect those of the Reserve Bank of New Zealand. A MATLAB function and some example code accompanying this article is available on my website https://sites.google.com/site/benjaminwongshijie. I thank Nathan Balke, Efrem Castelnuovo, Todd Clark, James Morley, Shaun Vahey, and Tugrul Vehbi for comments and discussion. I also thank Nathan Balke, Giovanni Caggiano, Efrem Castelnuovo, and Nicolas Groshenny for kindly making available code and data for the empirical examples. Any remaining errors, including that associated with using the code or data of the listed papers, are mine.

${ }^{\dagger}$ Reserve Bank of New Zealand. 2 The Terrace, Wellington 6011, New Zealand. Email: benjamin.wong@rbnz.govt.nz Tel: +64 44713957 


\section{Introduction}

Vector autoregression (VAR) models are workhorse models within empirical macroeconomics, and amongst it many applications, been used to understand causal influences of interesting shocks such as, inter alia, monetary policy, fiscal, and technology shocks. A widely reported output from VAR models is the historical decomposition. The idea is that all time series in the VAR can be fully decomposed into the contribution of the different shocks, and an exogenous component, which is referred to as the baseline projection. In other words, if we sum up the contribution of all the shocks at any time $t$, together with the baseline projection, we recover the original time series at time $t$. The historical decomposition is a counterfactual where one investigates how differently would variables have evolved if particular histories of shocks have instead occurred. Examples of prominent empirical applications using the historical decomposition as the primary tool to understand the importance of certain shocks during particular historical episodes are Burbidge and Harrison (1985) and Kilian (2009). A recent proliferation of nonlinear extensions to the standard VAR model naturally leads to demand to extend the historical decomposition into nonlinear settings. These nonlinear extensions include modeling threshold effects (e.g., see Auerbach and Gorodnichenko, 2012; Bachmann and Sims, 2012; Caggiano, Castelnuovo, and Groshenny, 2014; Fazzari, Morley, and Panovska, 2015), regime change (e.g. Sims and Zha, 2006), and smooth time-variation (e.g. Primiceri, 2005; Cogley and Sargent, 2005)

The main contribution of this article is to show how to conduct a historical decomposition for many existing popular nonlinear VAR extensions. There are two features of the historical decomposition introduced in this article that are noteworthy. First, I provide exact analytical solutions on how to conduct an exact historical decomposition applicable to many popular nonlinear VAR extensions. The exact analytical solution clears up any ambiguity of whether an exact historical decomposition in nonlinear VAR is even possible. For example, variants of the nonlinear version of the historical decomposition are often evaluated numerical, due to a lack of analytical solution (e.g. Balke, 2000; Kilian and Vigfusson, 2017). Moreover, the time series in question sometimes does not fully decompose, leaving the possibility of a remainder term. The provision of analytical solutions within this article clears this up ambiguity, and verifies that an exact historical decomposition for a number of popular nonlinear VAR models is indeed possible. Second, the historical decomposition calculated in the standard linear VAR is a nested case of the nonlinear VAR presented in this article. The nesting provides a lens to compare and contrast the historical decomposition in the nonlinear case relative to standard linear case. The nesting also reassures that the decomposition presented in this article is a natural counterpart to its linear companion. Indeed, because the linear case is nested, the exposition provides a framework to discuss how the inclusion of a constant in the nonlinear case acts as an 
implicit assumption of the model's steady state relative to the linear case. In particular, I discuss how such properties about the steady state may pose issues for interpretation, and if so, possible remedies.

The rest of the article is organized as follows. Section 2 presents the historical decomposition for nonlinear VAR models and how the presented approach compares to a standard linear VAR. Section 3 presents empirical examples and discussion. In particular, I revisit the Threshold VAR (TVAR) in the seminal work by Balke (2000), and the Smooth-Transition VAR (STVAR) that Caggiano, Castelnuovo, and Groshenny (2014) use to study the effect of uncertainty shocks. Through the lens of the empirical examples, I also provide discussion on the assumptions of the model's steady state, and how one might re-specify the model if needed. I will also provide a discussion and reconciliation to the Time-Varying Parameters VAR (TVP-VAR) introduced by Cogley and Sargent (2005) and Primiceri (2005), whose use has now proliferated. Section 4 concludes.

\section{The Historical Decomposition}

Consider the following VAR of order $p$

$$
\mathrm{y}_{\mathrm{t}}=\mu_{t}+\mathrm{A}_{1, \mathrm{t}} \mathbf{y}_{\mathrm{t}-1}+\ldots+\mathrm{A}_{\mathrm{p}, \mathrm{t}} \mathbf{y}_{\mathrm{t}-\mathrm{p}}+C_{t} \epsilon_{t}, \quad \epsilon_{t} \sim \mathbf{N}\left(\mathbf{0}, \mathbf{I}_{\mathbf{N}}\right)
$$

where $\mathbf{y}_{\mathbf{t}}=\left[y_{1, t}, y_{2, t}, \ldots, y_{N, t}\right]^{\prime}$ is a vector of $N$ variables, $\boldsymbol{\mu}_{\boldsymbol{t}}=\left[\mu_{1, t}, \mu_{2, t}, \ldots, \mu_{N, t}\right]^{\prime}$ is a vector of $N$ constants, and $\mathbf{A}_{\mathbf{i}, \mathbf{t}}$ is an $N \times N$ matrix of VAR coefficients for the $i^{\text {th }}$ lag. $\boldsymbol{\epsilon}_{\boldsymbol{t}}=\left[\epsilon_{1, t}, \epsilon_{2, t}, \ldots, \epsilon_{N, t}\right]^{\prime}$, is a vector of $N$ structural shocks which are assumed to be each normally and independently distributed and each with unit variance. The covariance matrix of the VAR is $\Sigma_{t}$ where $\Sigma_{t}=C_{t} C_{t}^{\prime}$, and the matrix $\boldsymbol{C}_{\boldsymbol{t}}$ maps the reduced form VAR residuals to the structural shocks, $\boldsymbol{\epsilon}_{\boldsymbol{t}}$. The notation is such that everything with a subscript $t$ can be time-varying. The exposition can be made time invariant by dropping all the $t$ subscripts from the constants, VAR coefficients, and covariance matrices.

It is more convenient to use the companion matrix for the exposition. We therefore rewrite Equation (1) as

$$
\mathrm{Z}_{\mathrm{t}}=\mathrm{H} \mu_{t}+\mathrm{A}_{\mathrm{t}} \mathrm{Z}_{\mathrm{t}-1}+\mathrm{H} C_{t} \epsilon_{t}
$$

where

$$
\mathbf{Z}_{\mathrm{t}}=\left[\begin{array}{c}
\mathbf{y}_{\mathbf{t}} \\
\vdots \\
\mathbf{y}_{\mathbf{t}-\mathbf{p}+\mathbf{1}}
\end{array}\right], \mathbf{A}_{\mathbf{t}}=\left[\begin{array}{ccc}
\mathbf{A}_{1, \mathbf{t}} & \cdots & \mathbf{A}_{\mathbf{p}, \mathbf{t}} \\
\mathbf{I}_{\mathbf{N}(\mathbf{p}-\mathbf{1})} & & \mathbf{0}_{\mathbf{N}(\mathbf{p}-\mathbf{1}) \times \mathbf{N}}
\end{array}\right], \mathbf{H}=\left[\begin{array}{c}
\mathbf{I}_{\mathbf{N}} \\
\mathbf{0}_{\mathbf{N}(\mathbf{p}-\mathbf{1}) \times \mathbf{N}}
\end{array}\right]
$$

Recursive substitution of Equation (2) yields, 


$$
\begin{aligned}
& \mathrm{Z}_{\mathrm{t}}=\mathrm{H} \mu_{t}+\mathrm{A}_{\mathrm{t}} \mathrm{Z}_{\mathrm{t}-1}+\mathrm{H} C_{t} \epsilon_{t} \\
& =\mathbf{H} \mu_{t}+\mathbf{A}_{\mathbf{t}}\left\{\mathbf{H} \mu_{t-1}+\mathbf{A}_{\mathbf{t}-1} \mathbf{Z}_{\mathbf{t}-2}+\mathbf{H} C_{t} \epsilon_{t-1}\right\}+\mathbf{H} C_{t} \epsilon_{t} \\
& =\mathrm{H} C_{t} \epsilon_{t}+\mathrm{A}_{\mathrm{t}} \mathrm{H} C_{t-1} \epsilon_{t-1}+\mathrm{A}_{\mathrm{t}}\left\{\mathrm{A}_{\mathrm{t}-1} \mathrm{Z}_{\mathrm{t}-2}\right\}+\mathrm{H} \mu_{t}+\mathrm{A}_{\mathrm{t}} \mathrm{H} \mu_{t-1} \\
& =\mathrm{H} C_{t} \epsilon_{t}+\mathrm{A}_{\mathrm{t}} \mathbf{H} C_{t-1} \epsilon_{t-1}+\left\{\prod_{\mathrm{i}=0}^{1} \mathrm{~A}_{\mathrm{t}-\mathrm{i}}\right\} \mathrm{Z}_{\mathrm{t}-2}+\mathbf{H} \mu_{t}+\mathrm{A}_{\mathrm{t}} \mathbf{H} \mu_{t-1} \\
& =\underbrace{\mathbf{H} C_{t} \epsilon_{t}+\sum_{\mathbf{j}=\mathbf{1}}^{\mathrm{t}-(\mathrm{p}+\mathbf{1})}\left\{\prod_{\mathbf{i}=\mathbf{0}}^{\mathrm{j}-\mathbf{1}} \mathrm{A}_{\mathrm{t}-\mathbf{i}}\right\} \mathrm{HC}_{\mathrm{t}-\mathrm{j}} \boldsymbol{\epsilon}_{t-j}}_{\text {Contribution from Shocks }}+ \\
& \underbrace{\left\{\prod_{k=0}^{t-(p+1)} \mathbf{A}_{\mathbf{t}-\mathbf{k}}\right\} \mathbf{Z}_{\mathbf{p}}}_{\text {Initial Conditions }}+\underbrace{\mathbf{H} \boldsymbol{\mu}_{\boldsymbol{t}}+\sum_{\mathbf{j}=\mathbf{1}}^{\mathbf{t}-(\mathbf{p}+\mathbf{1})}\left\{\prod_{\mathbf{i}=\mathbf{0}}^{\mathbf{j}-\mathbf{1}} \mathbf{A}_{\mathbf{t}-\mathbf{i}}\right\} \mathbf{H} \boldsymbol{\mu}_{\boldsymbol{t}-\mathbf{j}}}_{\text {Steady State Component }} .
\end{aligned}
$$

Equation (3) shows that the time series contained in $\mathbf{Z}_{\mathbf{t}}$, and thus by extension, $\mathbf{y}_{\mathbf{t}}$, are just linear functions of the history of structural shocks, $\left\{\boldsymbol{\epsilon}_{j}\right\}_{j=p+1}^{t}$, and an exogenous component, which will be referred to as the baseline projection. I will break up the baseline projection into two components. The first, termed "initial conditions", is due to the VAR taking the initial observations as given. ${ }^{1}$ The second component of the baseline projection is due to the inclusion of a constant in the VAR. I term this as the steady state component as it will turn out, this term is crucial for determining the model's steady state, a point we will return to later. Dropping all the $t$ subscripts from $\mathbf{A}_{\mathbf{t}}, \mathbf{C}_{\mathbf{t}}$, and $\boldsymbol{\mu}_{\boldsymbol{t}}$ implies a standard linear VAR with a constant covariance matrix with Equation (3) collapsing into

$$
\mathbf{Z}_{\mathbf{t}}=\underbrace{\sum_{j=0}^{t-(p+1)} \mathbf{A}^{\mathbf{j}} \mathbf{H C} \boldsymbol{\epsilon}_{\boldsymbol{t}-\boldsymbol{j}}}_{\text {Contribution from shocks }}+\underbrace{\underbrace{\mathbf{A}^{(\mathbf{t}-\mathbf{p})} \mathbf{Z}_{\mathbf{p}}}_{\text {Initial Conditions }}+\underbrace{\left\{\sum_{j=0}^{t-(p+1)} \mathbf{A}^{\mathbf{j}}\right\} \mathbf{H} \boldsymbol{\mu}}_{\text {Steady State Component }}}_{\text {Baseline Projection }} .
$$

It is straightforward to verify that Equation (4) is the historical decomposition associated with the standard linear VAR, and coincides with that found in work such as Burbidge and Harrison (1985). Together, Equations (3) and (4) imply four key implications for the historical decomposition for nonlinear models presented within this article.

\footnotetext{
${ }^{1}$ Note that $\mathbf{Z}_{\mathbf{p}}=\left[\begin{array}{llll}\mathbf{y}_{\mathbf{p}}{ }^{\prime}, & \mathbf{y}_{\mathbf{p}-\mathbf{1}^{\prime}}, & \ldots & \mathbf{y}_{\mathbf{1}}{ }^{\prime}\end{array}\right]^{\prime}$.
} 
Analytical and exact expression Equation (3) verifies that as long as the nonlinear VAR implies a locally linear VAR process for every time period $t$, this can be cast into the form of Equation (1), and the exact historical decomposition in Equation (3) applies. Many popular variants of nonlinear VAR models within the empirical macroeconomics literature can naturally be expressed as a linear VAR process that is local to $t$. These include the Threshold VAR (TVAR), Smooth-Transition VAR (STVAR), the MarkovSwitching VAR, and the Time-Varying Parameter VAR (TVP-VAR). We will revisit two of them as empirical examples in the following section. The analytical form also clears up ambiguity on whether it is possible to obtain an exact decomposition for nonlinear models. The extent of this ambiguity should not be understated. For example, both Balke (2000) and Kilian and Vigfusson (2017) suggest historical decomposition-like objects for their nonlinear VAR models, where they acknowledge the possibility of a remainder to account for an inexact decomposition. In the empirical exercise of Balke (2000), this remainder term is nontrivial. Baumeister and Peersman (2013) use simulation methods to construct nonlinear impulse response functions as inputs to obtain their historical decomposition. Because their method is simulation based, it becomes unclear whether their method produces exact and analytical results. Equation (3) clarifies that not only can one conduct an exact decomposition, the analytical form implies that simulation is not necessary. ${ }^{2}$

The linear VAR is nested Because Equation (4) coincides with the historical decomposition in a standard linear VAR, we can view the typical linear VAR as a special case of the result obtained in Equation (3). The natural implication is that the decomposition in the form of Equation (3) is a nonlinear analogue of the standard historical decomposition from a linear VAR, as the latter is just a special case. The partitioning of Equation (3) into components such as the baseline projection and the contribution of shocks means that we have a direct comparison to its linear counterpart in Equation (4), whose properties are probably better understood.

Implications for the Steady State From Equation (4), under stationarity, we observe that the baseline projection in the linear VAR converges to a vector of constants. As $t \rightarrow$ $\infty$, the initial conditions component converges to zero, and the steady state component, due to $\sum_{j=0}^{\infty} \mathbf{A}_{\mathbf{j}}$ being a geometric sum, converges to $\left[\mathbf{I}_{\mathbf{N p}}-\mathbf{A}\right]^{-\mathbf{1}} \mathbf{H} \boldsymbol{\mu}$. The baseline projection thus converges to $\left[\mathbf{I}_{\mathbf{N p}}-\mathbf{A}\right]^{-1} \mathbf{H} \boldsymbol{\mu}$, which is the unconditional mean of the time

\footnotetext{
${ }^{2} \mathrm{~A}$ caveat is we may not be able to use the insights from this discussion, at least off the shelf, to consider a historical decomposition with specific nonlinearities such as censoring of variables (see, e.g. Kilian and Vigfusson, 2011), or volatility in mean type effects as they may not possess the local linear VAR form as shown in Equation (1). Alternative nonlinear decompositions provided by Balke (2000) and Kilian and Vigfusson (2017) can be applied when confronted by these forms of nonlinearities, but similarly also implies that in such settings, we still lack analytical solutions, if any, to characterize the historical decomposition.
} 
series contained in $\mathbf{Z}_{\mathbf{t}}$. It is common to attach a steady state interpretation to the values which the VAR converges to (see, e.g. Villani, 2009). Such an interpretation is natural as these are values which the variables will converge to in the long run, in the absence of shocks. Under maximum likelihood, $\left[\mathbf{I}_{\mathbf{N p}}-\mathbf{A}\right]^{-\mathbf{1}} \mathbf{H} \boldsymbol{\mu}$ recovers the sample mean of the time series contained in $\mathbf{Z}_{\mathbf{t}}$. While the baseline projection converges towards a constant steady state in the linear case, this is not necessarily true with its nonlinear counterpart in Equation (3). While the initial conditions component will still to converge to zero given sequences of stationary VAR parameters, the inclusion of a constant also means that the time-variation in either or both the constant, or the VAR parameters implies a time-varying steady state. There is no guarantee that the potentially time-varying steady state component will be well behaved in the nonlinear case. We will revisit this issue in the empirical examples, and suggest empirical strategies to deal with the time-varying steady state, especially if it poses issues for interpretation.

The historical decomposition is always backward looking The historical decomposition is always backward looking, regardless whether the model is linear or nonlinear. In other words, the historical decomposition treats everything as observed. Therefore, possessing the estimates of the model's parameters and the history of structural shocks is sufficient information to calculate the historical decomposition. This can be seen by observing that all the terms of the righthand side of Equations (3) and (4) have a time subscript no greater than $t$. Contrast the idea of the historical decomposition being backward looking, relative to the impulse response function. The definition of the impulse response function at horizon $h$ is the difference between the forecast with and without a shock at time $t+h$, conditional on the information set before the shock is fed into the system at time $t$, or more formally,

$$
I R F^{h}=\mathbb{E}_{t}\left[\mathbf{y}_{\mathbf{t}+\mathbf{h}} \mid \Xi^{\mathbf{t}-\mathbf{1}}, \hat{\epsilon}_{\mathbf{j}, \mathbf{t}}\right]-\mathbb{E}_{\mathbf{t}}\left[\mathbf{y}_{\mathbf{t}+\mathbf{h}} \mid \Xi^{\mathbf{t}-\mathbf{1}}\right]
$$

where $\boldsymbol{\Xi}^{t-1}$ is the information set at time $t-1$, and $\hat{\epsilon}_{j, t}$ is the shock chosen by the researcher to be fed into the system at time $t$ in order to obtain the impulse response function. Nonlinear models often do not have an analytical solution for the impulse response function because this requires a forecast of the evolution of the model's parameters. Simulation based methods in the form of Monte Carlo integration, as proposed by Koop, Pesaran, and Potter (1996), are generally used to evaluate Equation (5) to obtain the impulse response function for nonlinear VAR models. A feature of linear models, is that an analytical solution does exist for Equation (5). This stems from the fact that the linear VAR coefficients are expected to remain the same. ${ }^{3}$ Because one does not require simulation methods to calculate both the historical decomposition and impulse response

\footnotetext{
${ }^{3}$ Accordingly, the solution for linear models when keeping the coefficients fixed and using the notation in Equation (4) is $I R F^{h}=\mathbf{H}^{\prime} \mathbf{A}^{\mathbf{h}} \mathbf{H} \mathbf{C} \hat{\epsilon}_{\mathbf{j}, \mathbf{t}}$.
} 
function from a linear VAR model, this may lead one to conclude that one requires simulation methods to calculate both objects for nonlinear VAR models. As this discussion has demonstrated, the latter notion is not true. Because the impulse response function is forward looking due to the expectation operator, and the historical decomposition is backward looking, one only requires simulation methods for calculating the former in nonlinear VAR models, but the analytical expression derived in this article implies simulation is not necessary for the latter. The viewing of the historical decomposition as backward looking is another way of understanding why simulation is unnecessary. Most simulation techniques for nonlinear variants of the historical decomposition numerically evaluates some version of Equation (5). By demonstrating the analytical solution of the historical decomposition in nonlinear VAR models is backward looking, we explicitly break the link to Equation (5), so avoiding the simulation step.

\section{$3 \quad$ Empirical Examples}

For the empirical examples, we revisit the TVAR by Balke (2000) and the STVAR by Caggiano, Castelnuovo, and Groshenny (2014). ${ }^{4}$ Note that both papers do not conduct a historical decomposition such as the one considered in this articles, though Balke (2000) does proposes a historical decomposition-like object, known as the change in the forecast function $(\mathrm{CFF})$, in order to understand the extent of which the nonlinearities matter. The CFF will provide a useful contrast to the historical decomposition presented in this article. I will also expand further on how one might interpret and deal with implicit assumptions regarding the steady state within the context of the two empirical examples.

\subsection{Threshold VAR}

Balke (2000) considered a TVAR of the following

$$
\mathbf{Y}_{\mathbf{t}}=\boldsymbol{\alpha}^{1}+\mathbf{B}^{1}(\mathbf{L}) \mathbf{Y}_{\mathbf{t}}+\left[\boldsymbol{\alpha}^{2}+\mathbf{B}^{2}(\mathbf{L}) \mathbf{Y}_{\mathbf{t}}\right] \mathbb{I}\left(\mathbf{s}_{\mathbf{t}-\mathbf{d}}>\delta\right)+\left[\mathbf{D}^{1}+\mathbf{D}^{2} \mathbb{I}\left(\mathbf{s}_{\mathbf{t}-1}>\delta\right)\right] \mathbf{U}_{\mathbf{t}}
$$

where $\mathbf{Y}_{\mathbf{t}}$ is a vector of four variables; annualized quarter on quarter real output growth and CPI inflation, the Federal Funds rate and the commercial paper-Treasury bill spread. $\mathbf{U}_{\mathbf{t}}$ are the structural residuals, and are assumed to be normally distributed and independent, each of unit variance. The lag polynomial $\mathbf{B}^{\mathbf{i}}(\mathbf{L})=\mathbf{B}_{1}^{\mathrm{i}} \mathbf{L}+\mathbf{B}_{2}^{\mathbf{i}} \mathbf{L}^{2}+\ldots+\mathbf{B}_{\mathbf{p}}^{\mathbf{i}} \mathbf{L}^{\mathbf{p}}$, $i \in\{1,2\}$, represents VAR parameters associated with the two regimes. The matrices $\mathbf{D}^{\mathbf{1}}$

\footnotetext{
${ }^{4}$ The original data is kindly provided by the authors. Every effort is made to keep to the notation in the original two papers as much as possible. Any notation differences are made mostly to avoid using similar notation introduced earlier in this article, and also to keep the flow of the exposition in the current article to be as smooth as possible.
} 
and $\mathbf{D}^{2}$ map the structural residuals to the reduced form residuals in both regimes. The function II takes on the value of 1 when the threshold variable $s_{t-d}$ exceeds the threshold value of $\delta$, and 0 otherwise. Because the matrices $\mathbf{D}$ implies a regime dependent mapping between the reduced form residuals and the structural residuals, this also implies regime specific covariance matrices. Balke identifies the four shocks using a cholesky ordering in the order that I have introduced the variables. This is an identification assumption and implies the $\mathbf{D}$ matrices will be lower triangular. I will therefore present results based on the four shocks, which, similar to the original article, labelled them output shock, price shock, federal fund rate shock and paper-bill shock. Like Balke, I will use a two quarter moving average of the interest rate spread between the four to six month commercial paper relative to a six month treasury bill as the threshold variable, lagged $d$ quarters. I will similarly take $d=1$, as per the original paper. The TVAR can thus be described as two locally linear VARs, whose dynamics can be described by the parameters with the superscript 1 if the threshold variable is below the threshold, and the additional terms kicking in when the threshold variable is above the threshold. The lag order, $p$ is set to 4, as per the original paper. The sample is 1959Q1 to 1997Q3.

After estimating Equation (6), to conduct the historical decomposition, we cast the following terms in the TVAR into those implied by Equation (1)

$$
\begin{aligned}
\mathbf{y}_{\mathbf{t}} & =\mathbf{Y}_{\mathbf{t}} \\
\boldsymbol{\mu}_{t} & = \begin{cases}\boldsymbol{\alpha}^{\mathbf{1}} & \text { if } s_{t-1} \leq \delta \\
\boldsymbol{\alpha}^{\mathbf{1}}+\boldsymbol{\alpha}^{2} & \text { if } s_{t-1}>\delta\end{cases} \\
\mathbf{A}_{\mathbf{i}, \mathbf{t}} & =\left\{\begin{array}{ll}
\mathbf{B}_{\mathbf{i}}^{1} & \text { if } s_{t-1} \leq \delta \\
\mathbf{B}_{\mathbf{i}}^{1}+\mathbf{B}_{\mathbf{i}}^{2} & \text { if } s_{t-1}>\delta
\end{array} \text { for } \mathrm{i} \in\{1,2, \ldots p\}\right. \\
\mathbf{C}_{\mathbf{t}} & = \begin{cases}\mathbf{D}^{1} & \text { if } s_{t-1} \leq \delta \\
\mathbf{D}^{1}+\mathbf{D}^{2} & \text { if } s_{t-1}>\delta\end{cases} \\
\boldsymbol{\epsilon}_{t} & =\mathbf{U}_{\mathbf{t}} .
\end{aligned}
$$

Figure 1 presents the threshold variable of the two quarter moving average of the paper-bill spread and the estimated threshold to present a flavour of the two credit regimes estimated by Balke (2000). The spread rises in recessions, and most of the observations in the high spread regime coincide with NBER recessions.

Balke (2000) proposes calculating the change in forecast function (CFF) as an alternative to the historical decomposition in nonlinear models. Using notation introduced in

\footnotetext{
${ }^{5}$ The details about the estimation are omitted in the interest of space as they are as per the original paper.
} 
Figure 1: Two Quarter Moving Average of the Four to Six month Commercial Paper and Six Month Treasury Bill Spread

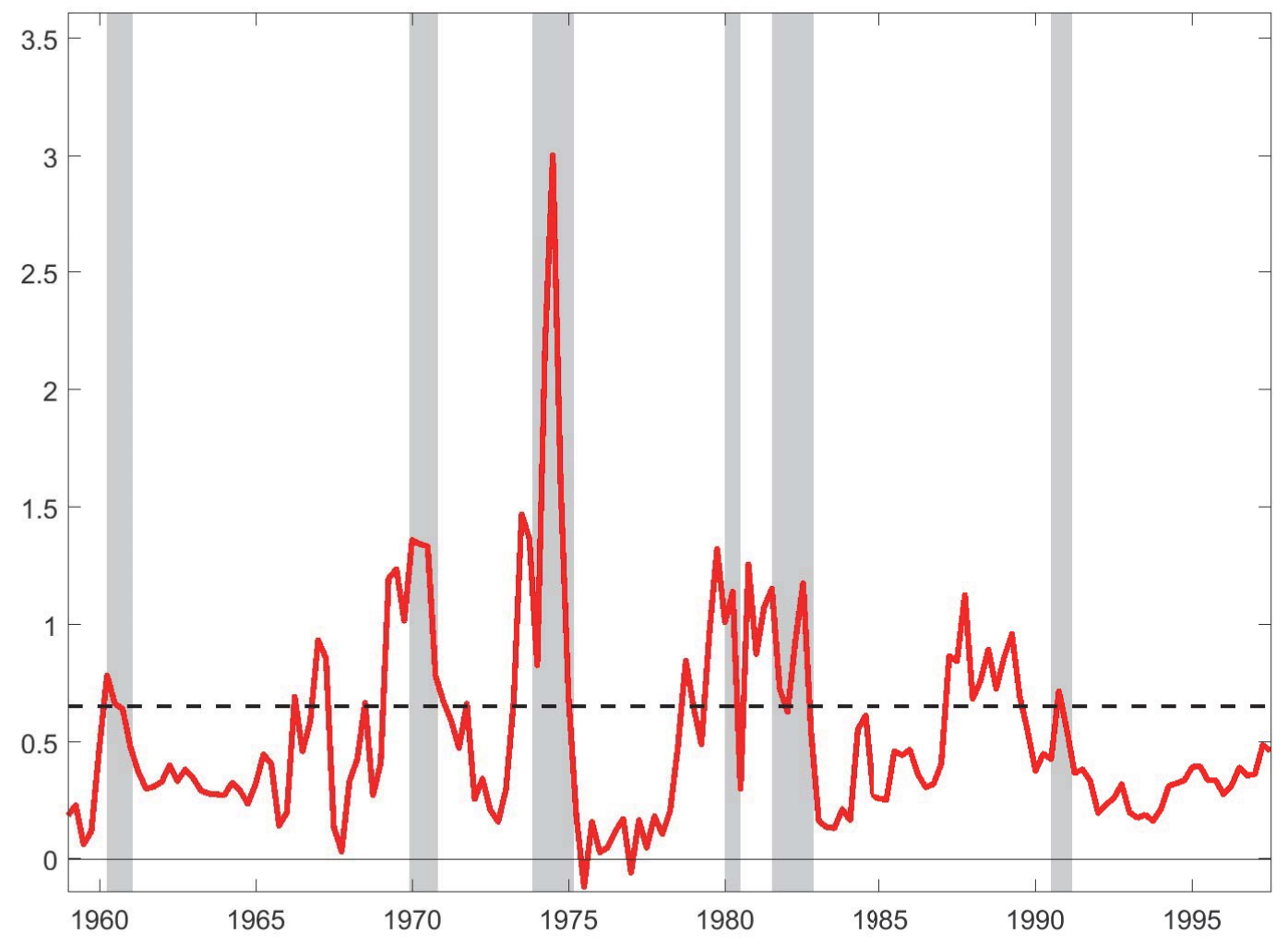

Notes: The shaded bars refers to the NBER dated recessions. Units in terms of percent per annum. Dotted line marks the estimated threshold, $\delta=0.65$.

Section 2, the CFF is defined as

$$
\operatorname{CFF}\left(\Xi^{t-1}, k, j\right)=\mathbb{E}_{t}\left[\mathbf{y}_{\mathbf{t}+\mathbf{k}} \mid \Xi^{\mathbf{t}-\mathbf{1}}, \epsilon_{\mathbf{j}, \mathbf{t}}, \epsilon_{\mathbf{j}, \mathbf{t}+\mathbf{1}}, \ldots, \epsilon_{\mathbf{j}, \mathbf{t}+\mathbf{k}}\right]-\mathbb{E}_{\mathbf{t}}\left[\mathbf{y}_{\mathbf{t}+\mathbf{k}} \mid \Xi^{\mathbf{t}-\mathbf{1}}\right]
$$

where $\epsilon_{j, t}$ is the realized $j^{\text {th }}$ structural shock in period $t$. There may be a remainder term if the unconditional forecast, $\mathbb{E}_{t}\left[\mathbf{y}_{\mathbf{t}+\mathbf{k}} \mid \boldsymbol{\Xi}^{\mathbf{t}-\mathbf{1}}\right]$, and the CFF does not add up to the time series, or more formally,

$$
\mathrm{R}_{m}\left(\Xi^{t-1}, k\right)=\mathbf{y}_{\mathbf{t}+\mathbf{k}}-\mathbb{E}_{\mathbf{t}}\left[\mathbf{y}_{\mathbf{t}+\mathbf{k}} \mid \Xi^{\mathbf{t}-\mathbf{1}}\right]-\sum_{\mathbf{j}=\mathbf{1}}^{\mathbf{N}} \operatorname{CFF}\left(\boldsymbol{\Xi}^{\mathbf{t}-\mathbf{1}}, \mathbf{k}, \mathbf{j}\right) .
$$

It is straightforward to verify under a linear VAR, the CFF can be analytically evaluated, and is identical to the historical decomposition for linear VAR models, as per the expression in Equation (4). Analogously, the remainder in a linear VAR model is zero by construction. The CFF generally does not have an analytical form in nonlinear models, 
and is often evaluated numerically using Monte Carlo integration, such as Koop, Pesaran, and Potter (1996). In nonlinear models, the remainder emerges because the nonliearity causes the contributions of shocks and the forecast to not add up. The remainder can thus be interpreted as the extent that the nonlinearity interacts with the shocks.

Figure 2: Historical Decomposition and Change in Forecast Function of Output Growth from TVAR
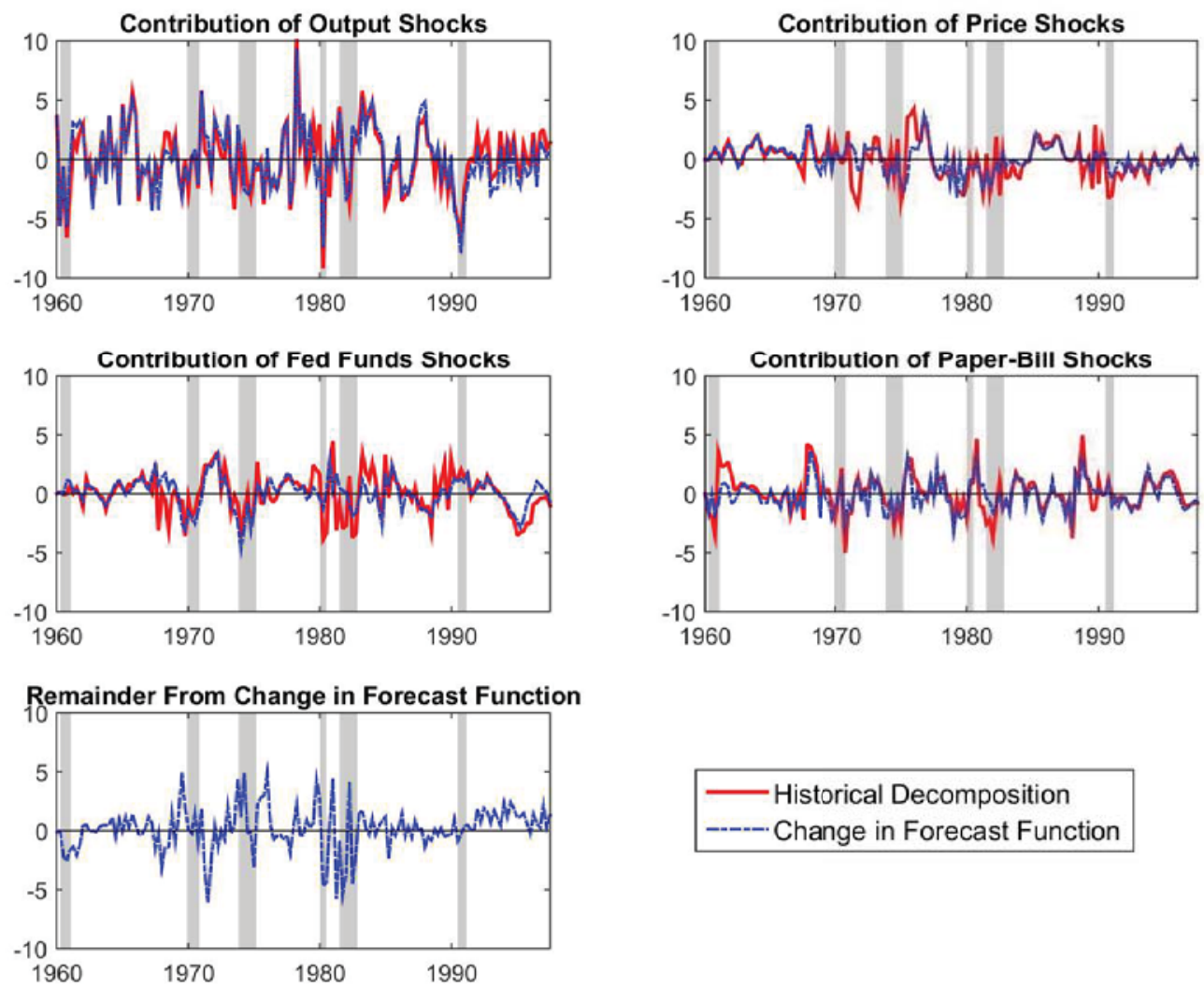

Notes: The shaded bars refers to the NBER dated recessions. The contribution of shocks in the historical decomposition are to deviation from the baseline projection. Units are in terms of annualized quarter on quarter real GDP growth.

Figure (2) presents the historical decomposition of output growth from the TVAR to the contribution of the four identified shocks. I also plot the CFF and its associated remainder. ${ }^{6}$

\footnotetext{
${ }^{6}$ Balke (2000) uses a horizon of 12 quarters to compute the CFF in his original paper. I use the entire sequence of shocks as this is the only construction where in a linear VAR model, the CFF is equivalent to the historical decomposition and the remainder from the CFF is zero. In this respect, I am evaluating the $\mathrm{CFF}$ in a manner much closer to the conditional structural decomposition by Kilian and Vigfusson (2017). This is to ensure that any remainder calculated in the empirical example is due to the nonlinearity interacting with the shock, and rules out the possibility that the remainder may be picking up the effect of a shock that has a high level of persistence. Note though that if the model's propagation mechanism is not extremely persistent, choosing a sufficiently long horizon, such as 12 quarters, may
} 
While there are many similarities between the historical decomposition and the CFF, the divergences between the two are most prevalent during or around recessions. This is not surprising as the threshold, which is the source of the nonlinearity, tends to kick in during recessions (see Figure 1). This can also be seen by observing the remainder term evidencing its largest variations during and around recessions, which can be interpreted as when the nonlinearity is being particularly relevant in propagating shocks.

Even if the remainder term in the CFF is useful in understanding when the nonlinearity is particularly relevant for propagating shocks, it does not decompose these fluctuations induced by the nonlinearity to contributions by any particular shocks. In other words, the remainder acts as a catch-all term which groups all variation due to the nonlinearity binding, but does not attribute this catch-all variation to any of the shocks. This is where the historical decomposition introduced in this article aids in providing such an interpretation.

We can reconcile much of the remainder term to divergences between the historical decomposition and the CFF. The greatest negative contribution in the remainder is just after the 1969-70 recession and the twin recession in the early 1980s. The historical decomposition reconciles the large negative remainder in the 1969-70 recession with the $\mathrm{CFF}$ being unable to account for price shocks. The historical decomposition attributes the negative contribution in the remainder during the twin recessions as a mix of Fed Fund rate and paper-bill shocks. We can also observe that the positive contribution to the remainder after the 1973-75 recession is attributed to price shocks by the historical decomposition. The persistent positive contribution of the remainder at the end of the sample can largely be attributed to the CFF being unable to attribute these to output shocks, which the historical decomposition does.

In summary, we can reconcile most of the remainder we obtain from the remainder of the CFF to contributions that the exact historical decomposition introduced in this article is able to pick out. While the CFF is unable to fully decompose the contribution of shocks within the TVAR, unlike the historical decomposition, the preceding analysis suggests that the remainder term may be a useful complement to the historical decomposition. This is especially so when the CFF is used in conjunction to understand whether the propagation of specific shocks interacting with the model's nonlinear features are relevant during particular historical episodes.

\subsection{Modelling the Steady State}

A closer observation of Figure 2 reveals that while the historical decomposition can accounts for much of the fluctuations in the remainder, it does not account for all the fluctuations in the remainder. It turns out that understanding all of the fluctuations provide a sufficiently good approximation. 
through the lens of the shocks is linked to the implicit assumptions one makes of the steady state of the model, an issue motivated earlier as being related to the handling the inclusion of a constant in the nonlinear VAR system.

Figure 3: Baseline Projection From Estimated TVAR

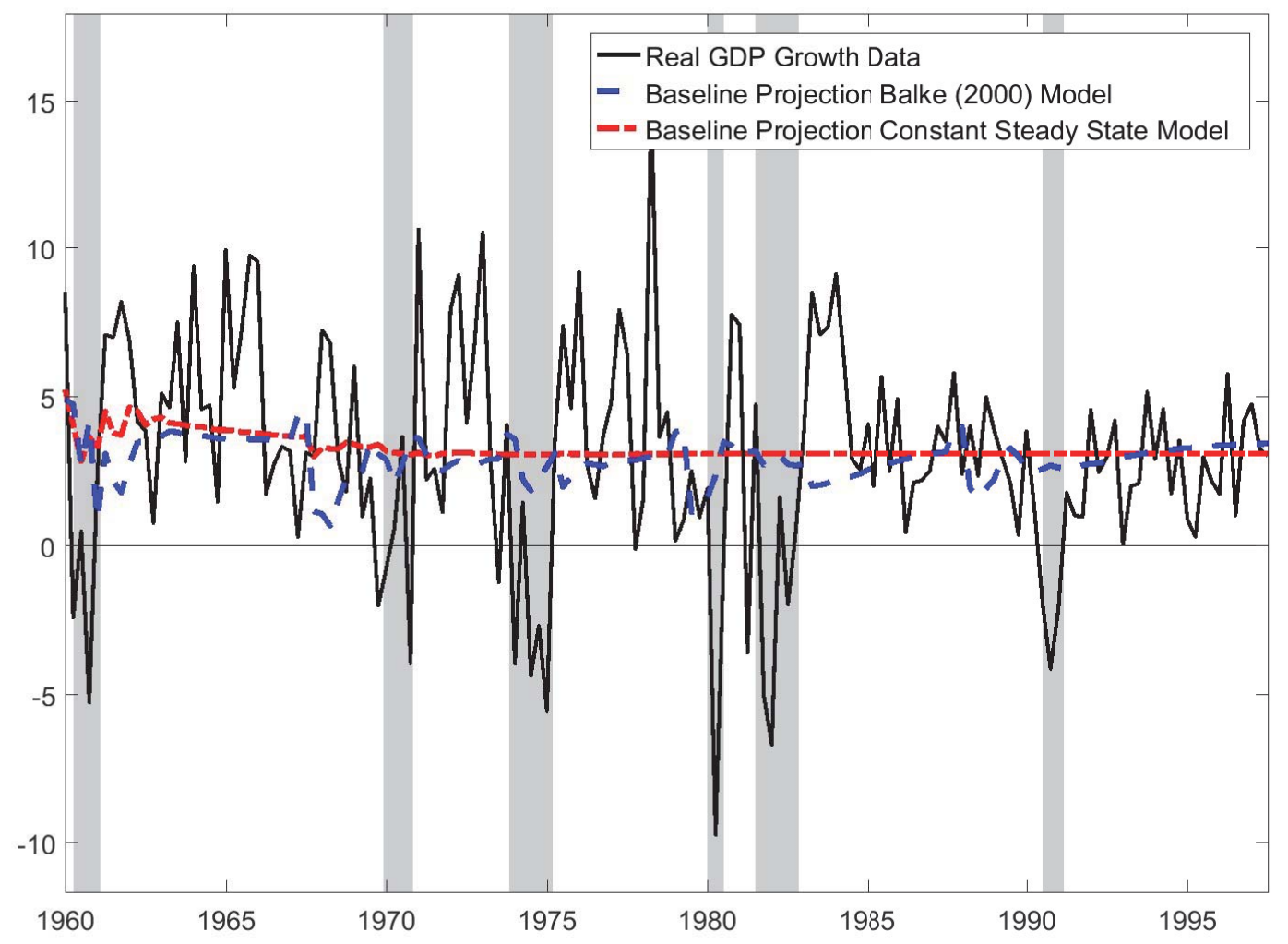

Notes: The shaded bars refers to the NBER dated recessions. Units are in terms of annualized quarter on quarter real GDP growth. Constant steady state is implemented by demeaning relative to sample mean and subsequently estimating the TVAR without a constant. The Balke (2000) model is the benchmark model considered in Figure 2.

To fix ideas, I first discuss the baseline projection from the original TVAR model estimated through Equation (6), which contains a regime specific constant. This is shown as the dotted line in Figure 3. The baseline projection "bounces around", and does not appear to settle. As discussed, and can be seen through observing Equation (3), this is because the steady state component does not asymptote to a vector of constants, unlike the linear case. In other words, the TVAR implies a time-varying steady state, and the baseline projection is constantly adjusting towards its new steady state. In our example, the baseline projection never reaches any of its implied steady states, because it has to constantly adjust towards another new steady state even before it gets close to the previous steady state it was adjusting towards. This feature leads to the observation of 
the baseline projection "bouncing around" and never being able to settle.

We also observe the baseline projection falling in recessions. This reflects that the steady state growth is much lower in the recession regime. The changing baseline projection can account for all remaining discrepancies between the contribution of the shocks and the fluctuation in output growth from the historical decomposition.

\section{Economic Interpretation of Steady State Component in Baseline Projection}

On their own, the historical decomposition performed is just a mechanical application of the expression in Equation (3), and yields no conclusion about whether an implicit time-varying steady state is appropriate. Given the historical decomposition is used to understand historical fluctuations in the economic data, a relevant consideration would be the economic interpretation one attaches to the bouncing steady state. From this perspective, the "bouncing" baseline projection presents two challenges for economic interpretation. First, because there is an exogenous shift in the steady state that is not linked to the economic shocks within the model, it becomes less satisfactory as an economic interpretation to attribute changes to a shifting steady state, especially one that is anything but steady or stable. Note that the historical decomposition in a linear VAR model has traditionally been used to explain all the fluctuation in the data through the contribution of the shocks, and one can only reliably do so if the baseline projection is well-behaved, in the sense of adjusting mechanically towards its (constant) steady state. That nonlinear VAR models already feature a baseline projection that does not adjust mechanically towards a constant steady state means that as a minimum, one should exercise caution when reconciling the historical decomposition obtained through linear and nonlinear VAR models. Second, few, if any, economic models imply a constantly evolving steady state, especially one that could differ between expansions and recessions. For example, one needs to put forward a theory why trend inflation is lower in a recession, or the natural rate of unemployment is higher in a recession, as these are objects related to some notion of the "steady state" and economic theory does not suggest these "steady state" values shift in recessions relative to expansions. If anything, economic theory is more likely to assert these steady state values are more time invariant, and fluctuations away from these steady states in recessions and expansions are more likely to be regarded as "cyclical", and are expected to reverse to steady states once these "cycles" have dissipated.

Implementing a Constant Steady State The preceding discussion suggest there is at least a need to be able to model a constant steady state in nonlinear VAR models, especially if one wanted to allow for features such as, a constant natural rate of unemployment or the model implying some "trend level" of output growth in the long run as a steady state. That is, there is some economic rationale for why one might want to impose a constant steady state, at least within certain contexts. If so, Equation (3) once 
again provides some clues on how one could respecify a nonlinear VAR model to imply a constant steady state. One solution is to estimate the model in terms of deviations from steady state and not including a constant. This effectively models Equation (2) as

$$
\left[\mathbf{Z}_{\mathrm{t}}-\overline{\mathbf{Z}}\right]=\mathbf{A}_{\mathbf{t}}\left[\mathbf{Z}_{\mathrm{t}-1}-\overline{\mathbf{Z}}\right]+\mathbf{H} C_{t} \epsilon_{t}
$$

where $\overline{\mathbf{Z}}$ contains the steady state values of the variables contained in the vector $\mathbf{Z}_{\mathbf{t}}$. It is straightforward to see that if the nonlinear VAR was implement in the form of Equation (9), the steady state component for $\mathbf{Z}_{\mathbf{t}}-\overline{\mathbf{Z}}$ is zero by construction (due to setting $\boldsymbol{\mu}_{\boldsymbol{t}}=0$ ). The initial conditions component is expected to adjust towards zero under stationarity, and once the initial conditions components decays, the baseline projection for $\mathbf{Z}_{\mathbf{t}}$ is by construction, $\overline{\mathbf{Z}} .^{7}$ One could set $\overline{\mathbf{Z}}$ by appealing to extraneous estimates. In some cases, this could be obvious. $\overline{\mathbf{Z}}$ could be the natural rate of unemployment, the inflation target or the natural rate of interest. ${ }^{8}$ In my empirical example, I will just take the sample mean as the steady state value. I thus impose a constant steady state with the TVAR by demeaning relative to the sample mean and estimating the TVAR without a constant. Figure(3) shows the baseline projection of output growth from this modified specification, relative to the TVAR estimated earlier. As expected, the baseline projection is now roughly a constant, reflecting the imposition of a constant steady state.

Figure 4 plots the historical decomposition from the modified model which imposes a constant steady state, relative to the ones estimated in Figure (2). It appears inference about the contribution of output shocks are not overly affected by the adjusting the model's steady state. However, the model imposing a constant steady state now implies that the Fed fund rate and paper-bill shocks contributed more negatively to output growth during the twin recessions of the early 1980s. Moreover, the contributions from the shocks between the two specifications differ most during recessions, reflecting that what was attributed to a shifting steady state in the baseline model, is now accounted for by the underlying identified shocks within the model.

The purpose of this discussion is to emphasize that the inclusion of a constant in nonlinear models is not an innocuous choice, and one that could be easily overlooked. If one wishes to understand the fluctuations of the data through the lens of the underlying shocks by using a historical decomposition from the nonlinear model, then one needs to make a choice of whether modelling a constant is appropriate. The preceding discussion

\footnotetext{
${ }^{7}$ Note in a linear VAR, if we set $\overline{\mathbf{Z}}$ to the mean of the time series, the estimates of $\mathbf{A}$ are identical to estimating the VAR on raw data with a constant (e.g., see Lütkepohl, 2007, pg. 26 on a VAR in "mean-adjusted" form). Therefore, demeaning the data and estimating the VAR without a constant with the linear VAR does not change the model in any way.

${ }^{8}$ This discussion also implies that $\overline{\mathbf{Z}}$ does not necessarily need to be constant across the whole sample. For example, if one regarded the natural rate of interest has fallen, one is always free to adjust relative towards such an estimate given there is an economic rationale in doing so. The only issue to note is that this estimate is extraneous, and not estimated within the model.
} 
Figure 4: Historical Decomposition of Output Growth from TVAR
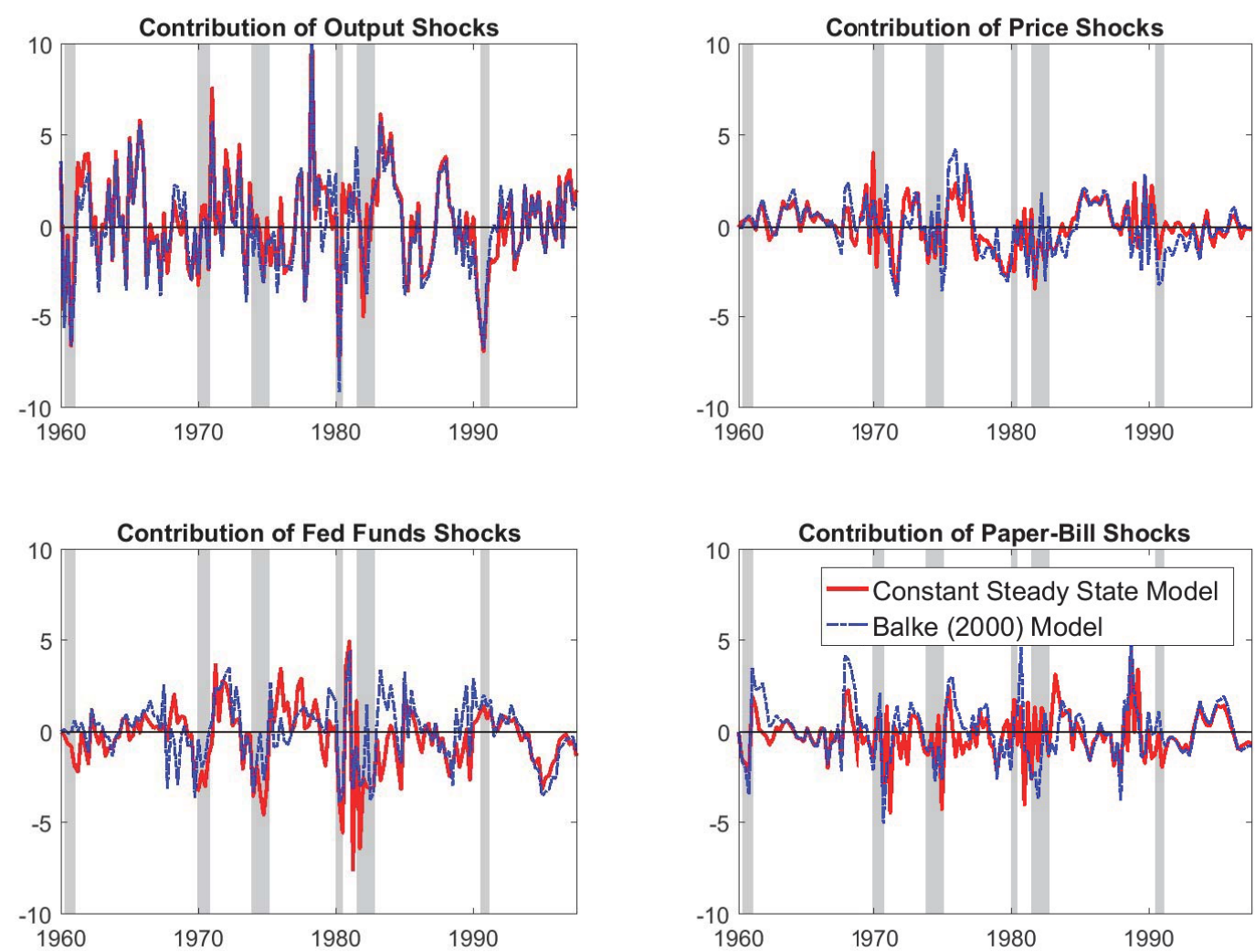

Notes: The shaded bars refers to the NBER dated recessions. The contribution of shocks in the historical decomposition are to deviation from the baseline projection. Units are in terms of annualized quarter on quarter real GDP growth. Constant steady state is implemented by demeaning relative to sample mean and subsequently estimating the TVAR without a constant. The Balke (2000) model is the benchmark model considered in Figure 2.

shows such a choice may have first order implications for the underlying assumptions regarding the model's steady state.

\subsection{Smooth-Transition VAR}

I will use the work by Caggiano, Castelnuovo, and Groshenny (2014), henceforth CCG, to illustrate the STVAR application. CCG investigate the impact of uncertainty shocks in a four variable VAR consisting of the VIX, U.S. unemployment, U.S. inflation, and the Federal Funds Rate. They estimated both a standard linear VAR and an STVAR with the four variables for a sample from 1963Q3 to 2012Q2. The use of a linear VAR will be 
a useful comparison, especially given the approach in this article nests the linear VAR. ${ }^{9}$ Their STVAR can be described as follows:

$$
\begin{aligned}
X_{t} & =\psi+F\left(w_{t-1}\right) \Phi_{R}(L) X_{t}+\left(1-F\left(w_{t-1}\right)\right) \Phi_{N R}(L) X_{t}+\zeta_{t} \\
\zeta_{t} & \sim N\left(0, \Gamma_{t}\right) \\
\Gamma_{t} & =F\left(w_{t-1}\right) \Gamma_{R}+\left(1-F\left(w_{t-1}\right)\right) \Gamma_{N R} \\
F\left(y_{t}\right) & =\exp \left(-\gamma w_{t}\right) /\left(1+\exp \left(-\gamma w_{t}\right)\right), \gamma>0, w_{t} \sim N(0,1)
\end{aligned}
$$

where $X_{t}$ is the vector of their four variables, and $\Phi_{i}(L)$ is a lag polynomial $\Phi_{i}(L)=$ $\Phi_{i}^{1} L+\Phi_{i}^{2} L^{2}+\ldots+\Phi_{i}^{p} L^{p}$, where $i \in\{R, N R\}$. We can think of the STVAR as being a linear combination of two VARs, where one describes the dynamics of the time series in recessions, and is governed by the parameters in the lag polynomial $\Phi_{R}(L)$ with covariance matrix, $\Gamma_{R}$, and another which describes the dynamics in expansions, governed by the parameters, $\Phi_{N R}(L)$ and $\Gamma_{N R}$. The transition function, $F\left(w_{t}\right)$, is described by a transition variable $w_{t}$, which they have taken to be a moving seven quarter growth rate of real GDP, normalized to mean zero and unit variance. The function $F($.$) estimates the probability$ of being in a recession, and the STVAR at time $t$ combines the two linear VARs based on the recession probability. The parameter $\gamma$, is set to 1.75 , to correspond with recessions occurring in about $17 \%$ in their sample. $\psi$ is a constant. CCG identified uncertainty shocks by ordering the VIX first in a Cholesky decomposition. A key result in CCG's paper is that the estimated effect of uncertainty shocks on variables such an unemployment, the Fed fund rate and inflation are greater in recessions within the STVAR relative to the estimate one obtains from a linear VAR.

Post-estimation, the STVAR characterized using Equations (10) to (13) can be cast into the form described in Equation (1) as

$$
\begin{aligned}
\mathbf{y}_{\mathbf{t}} & =X_{t} \\
\boldsymbol{\mu}_{\boldsymbol{t}} & =\psi \\
\mathbf{A}_{\mathbf{i}, \mathbf{t}} & =F\left(w_{t-1}\right) \Phi_{R}^{i}+\left(1-F\left(w_{t-1}\right)\right) \Phi_{N R}^{i} \\
\mathbf{C}_{\mathbf{t}} & =\operatorname{chol}\left[F\left(w_{t-1}\right) \Gamma_{R}+\left(1-F\left(w_{t-1}\right)\right) \Gamma_{N R}\right] \\
\boldsymbol{\epsilon}_{\boldsymbol{t}} & =\left\{\operatorname{chol}\left[F\left(w_{t-1}\right) \Gamma_{R}+\left(1-F\left(w_{t-1}\right)\right) \Gamma_{N R}\right]\right\}^{-1} \zeta_{t} .
\end{aligned}
$$

where $\operatorname{chol}($.$) applies the cholesky decomposition. Figure 5$ presents the historical decomposition of the various variables to an uncertainty shock using both the STVAR and

\footnotetext{
${ }^{9}$ Note once again that CCG do not conduct a historical decomposition and only do impulse response function analysis in their paper. Any presented historical decomposition is calculated by me. All the calculations uses three lags, as per CCG's paper.
} 
Figure 5: Role of Uncertainty Shocks and Estimated Baseline Projection
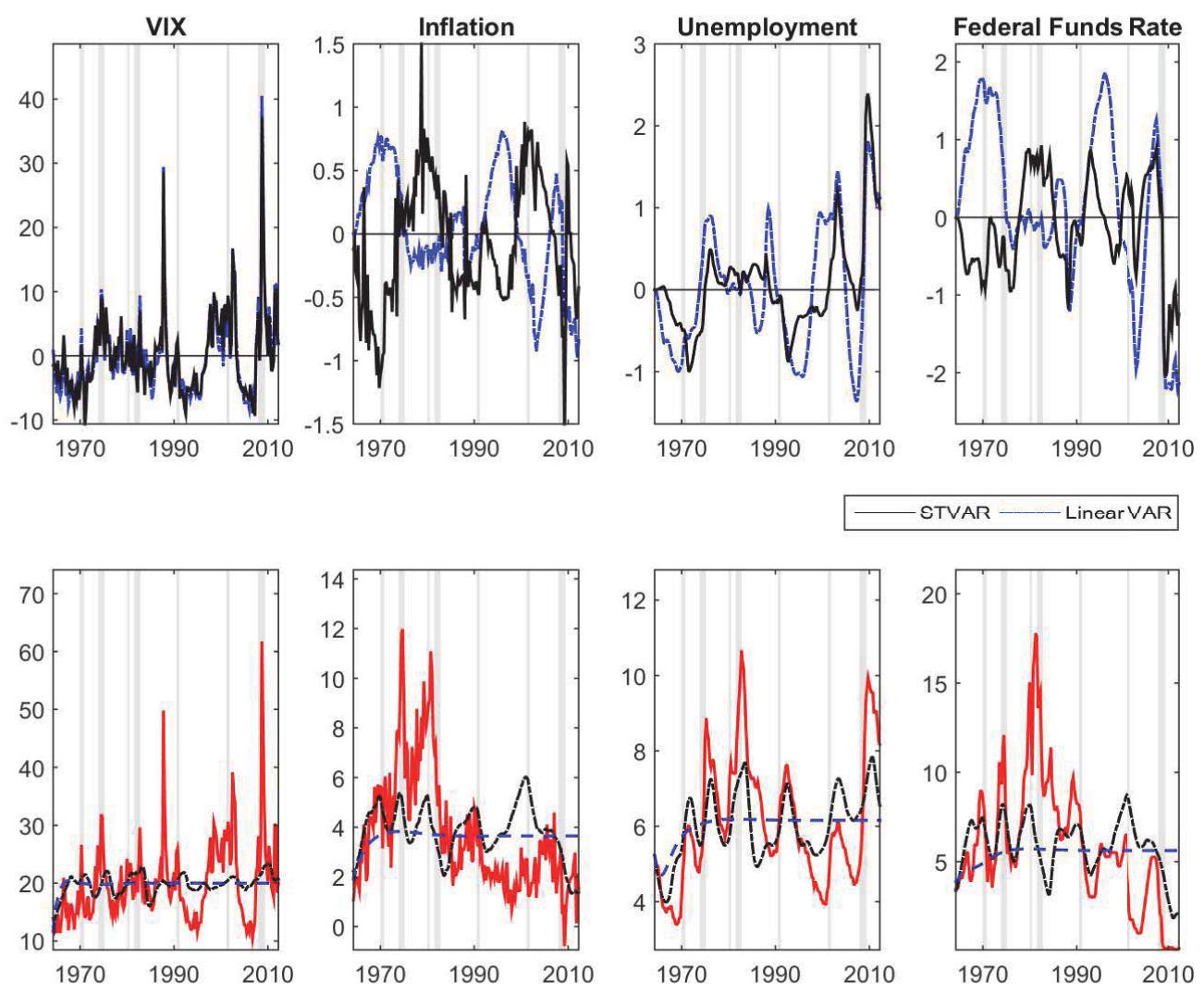

Baseline Projection, STVAR - - - Baseline Projection, Linear VAR

Notes: Top panel are estimated historical decompositions. Bottom panel presents estimated baseline projection from respective models. The historical decomposition measures the contribution of uncertainty shocks to the variables from their respective baseline projection. The shaded bars refers to the NBER dated recessions. VIX is in the units of the raw VIX index. Inflation is in units of quarter on quarter annualized rate. Unemployment is in units of percentage points. Federal Funds rates is in units of percent per annum.

the linear VAR, with the former calculated using Equation (3). The baseline projection of the associated variables are also presented. While the historical decomposition appears reasonable from the perspective of our understanding of history, the contribution of uncertainty shocks with the linear VAR appears to have more variation than the STVAR, especially around recession. This seems puzzling given a core result of CCG is that the responses of variables are more pronounced in recessions. Once again, an inspection of the baseline projection suggest that it is the steady state component that accounts for some of the variation of the variables in recessions for the STVAR. In particular, the baseline projection for unemployment rises in recessions, thus accounting for part of the variation in unemployment around recessions. 
Figure 6: Historical Decomposition of Uncertainty Shock Adjusting for Constant Steady State
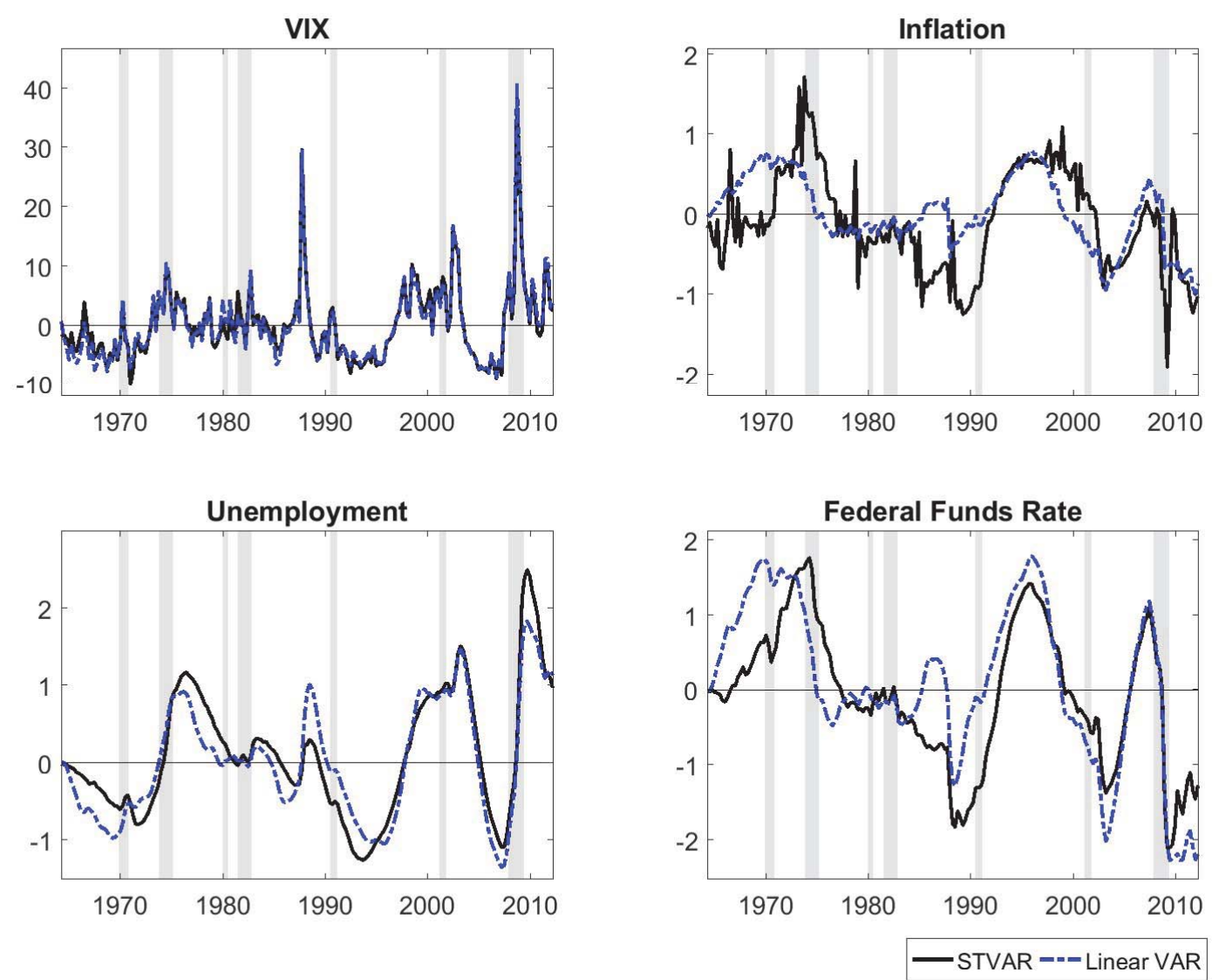

Notes: The historical decomposition measures the contribution of uncertainty shocks to the variables from their respective baseline projection. The shaded bars refers to the NBER dated recessions. VIX is in the units of the raw VIX index. Inflation is in units of quarter on quarter annualized rate. Unemployment is in units of percentage points. Federal Funds rates is in units of percent per annum.

We can once again set a time-invariant steady state. Taking the sample mean as the steady state, I estimate the STVAR after demeaning all the variables by their sample means, and subsequently re-do the historical decomposition. Note that this does not change the parameter estimates of the linear VAR as the latter approach is just estimating based on its "mean adjusted" form. Figure (6) presents the historical decomposition from the linear VAR and STVAR estimated using demeaned data and dropping the constant. ${ }^{10}$ The counterintuitive results of Figure 5 are now overturned. Because we have allowed for the same steady state in both the linear and STVAR, the baseline projection no longer plays any role in comparing both sets of historical decompositions. The demeaned specification also appeals to economic intuition that the steady state values of all the

\footnotetext{
${ }^{10}$ The key result of CCG with the impulse response analysis still holds in this specification, with the response to uncertainty shocks larger in recessions relative to the linear model.
} 
variables in the VAR should be invariant to the state of the business cycle. We now observe the historical decomposition from the STVAR witnesses more fluctuation than the linear VAR. In particular, we observe that a greater proportion of fluctuations in unemployment during recessions can now be explained by uncertainty shocks in the STVAR relative to the linear VAR, especially during the Great Recession and the 1973-75 recession.

In sum, once one appeals to economic intuition and models the steady state values of variables to be invariant to the state of the business cycle, the historical decomposition reproduces the same conclusions as CCG. Uncertainty shocks exert a greater effect in recessions relative to expansions, and seems particularly relevant for understanding unemployment dynamics during the Great Recession.

\subsection{Time-Varying Parameter Models}

Given its proliferation, it is appropriate to comment briefly on TVP-VAR models, even in the absence of an explicit empirical example. Use of the flexible TVP-VAR model, in the spirit of Cogley and Sargent (2005) and Primiceri (2005), has proliferated and used to studying a wide variety of problems such as the time-varying impact of technology shocks (e.g. Gambetti and Galí, 2009), monetary policy shocks (e.g. Canova and Gambetti, 2009) and oil price shocks (e.g. Clark and Terry, 2010; Baumeister and Peersman, 2013), among others. A typical specification models all parameters as evolving as driftless random walks. Concentrating on just the VAR coefficients, this involves a specification such as $\mathbf{A}_{\mathbf{t}+\mathbf{1}}=\mathbf{A}_{\mathbf{t}}+\vartheta_{\mathbf{t}}$, where $\vartheta_{t}$ are mean zero, and possibly correlated, innovations to the VAR coefficients. The historical decomposition described in Equation (3) only decomposes the shocks to the VAR equation, but not of the innovation to the VAR coefficients. Decomposing the role of the shocks to the VAR innovations is not straightforward, at least with the issues discussed in the article, as this involves the interaction of the innovations to the VAR coefficients and the VAR structural shocks. Certainly, the decomposition presented in Equation (3) can be applied to the TVP-VAR case, if one takes the sequence of $\left\{\mathbf{A}_{\mathbf{j}}\right\}_{j=1}^{t}$ as given, and just work out the contribution of the VAR structural shocks, which is effectively the approach taken by Clark and Terry (2010) and Clark and Davig (2011). ${ }^{11}$ Nonetheless, the same considerations about the inclusion of a constant remain, and needs to be assessed for it appropriateness for the particular empirical application in question. In particular, the inclusion of a constant assumes that the baseline projection will reflect a steady state which is driven by an underlying driftless random walk process.

\footnotetext{
${ }^{11}$ The two listed papers work out the forecast period by period, adding in the VAR shocks one at a time. The calculations presented in Equation (3) effectively does an identical calculation as long as we similarly take the history of VAR coefficients as given, and not attempt to disentangle the innovations to the VAR coefficients.
} 


\section{Conclusion}

The purpose of this article is to clarify how one can conduct a historical decomposition for nonlinear structural vector autoregression models. The discussion applies for a wide class of popular nonlinear structural vector autoregression models, such as the TVAR, STVAR, TVP-VAR, and Markov-Switching VAR, to name a few. The historical decomposition presented in this article is analytical, and thus does not require simulation techniques. The linear case is also nested within the nonlinear VAR case. Finally, I note that the choice of modelling a constant in nonlinear VAR applications is not innocuous, due to implicit assumptions about the steady state of the model. The implicit assumptions of the steady state seems a particularly relevant issue that applied researchers should at least be aware, and assessed for its appropriateness when making modelling choices.

\section{References}

Auerbach, A. J., And Y. Gorodnichenko (2012): "Measuring the Output Responses to Fiscal Policy," American Economic Journal: Economic Policy, 4(2), 1-27.

Bachmann, R., And E. R. Sims (2012): "Confidence and the transmission of government spending shocks," Journal of Monetary Economics, 59(3), 235-249.

Balke, N. S. (2000): "Credit and Economic Activity: Credit Regimes and Nonlinear Propagation of Shocks," The Review of Economics and Statistics, 82(2), 344-349.

Baumeister, C., And G. Peersman (2013): "Time-Varying Effects of Oil Supply Shocks on the US Economy," American Economic Journal: Macroeconomics, 5(4), 1-28.

Burbidge, J., AND A. HARrison (1985): "An historical decomposition of the great depression to determine the role of money," Journal of Monetary Economics, 16(1), 45-54.

Caggiano, G., E. Castelnuovo, and N. Groshenny (2014): "Uncertainty shocks and unemployment dynamics in U.S. recessions," Journal of Monetary Economics, 67(C), 78-92.

Canova, F., And L. Gambetti (2009): "Structural changes in the US economy: Is there a role for monetary policy?," Journal of Economic Dynamics and Control, 33(2), 477-490.

Clark, T. E., AND T. Davig (2011): "Decomposing the Declining Volatility of Long-Term Inflation Expectations," Journal of Economic Dynamics and Control, 35(7), 981-999.

Clark, T. E., And S. J. Terry (2010): "Time Variation in the Inflation Passthrough of Energy Prices," Journal of Money, Credit and Banking, 42(7), 1419-1433.

Cogley, T., And T. J. Sargent (2005): "Drift and Volatilities: Monetary Policies and Outcomes in the Post WWII U.S," Review of Economic Dynamics, 8(2), 262-302.

Fazzari, S. M., J. Morley, And I. Panovska (2015): "State-dependent effects of fiscal policy," Studies in Nonlinear Dynamics \& Econometrics, 19(3), 285-315. 
Gambetti, L., And J. Galí (2009): "On the Sources of the Great Moderation," American Economic Journal: Macroeconomics, 1(1), 26-57.

Kilian, L. (2009): "Not All Oil Price Shocks Are Alike: Disentangling Demand and Supply Shocks in the Crude Oil Market," American Economic Review, 99(3), 1053-69.

Kilian, L., AND R. J. Vigfusson (2011): "Are the responses of the U.S. economy asymmetric in energy price increases and decreases?," Quantitative Economics, 2(3), 419-453.

(2017): "The role of oil price shocks in causing U.S. recessions," Journal of Money, Credit and Banking, forthcoming.

Koop, G., M. H. Pesaran, And S. M. Potter (1996): "Impulse response analysis in nonlinear multivariate models," Journal of Econometrics, 74(1), 119-147.

Lütкepohl, H. (2007): New Introduction to Multiple Time Series Analysis. Springer Berlin Heidelberg.

Primiceri, G. E. (2005): "Time Varying Structural Vector Autoregressions and Monetary Policy," Review of Economic Studies, 72(3), 821-852.

Sims, C. A., AND T. Zha (2006): "Were There Regime Switches in U.S. Monetary Policy?," American Economic Review, 96(1), 54-81.

Villani, M. (2009): "Steady-state priors for vector autoregressions," Journal of Applied Econometrics, $24(4), 630-650$. 